\title{
Assessment of Pneumatic Balloon Dilation in Patients with Symptomatic Relapse after Failed Heller Myotomy: A Single Center Experience
}

\author{
Mohammad Amani ${ }^{1 \dagger}$, Narges Fazlollahi ${ }^{1 \dagger}$, Shapour Shirani ${ }^{2}$, Reza Malekzadeh ${ }^{1}$, Javad Mikaeli ${ }^{1 *}$ \\ 1. Digestive Disease Research Institute, \\ Shariati Hospital, Tehran University of \\ Medical Sciences, Tehran, Iran \\ 2. Department of Radiology, Tehran Heart \\ Center, Tehran University of Medical Sci- \\ ences, Tehran, Iran \\ $\dagger$ These two authors contributed equally to this \\ paper

\section{ABSTRACT}

\section{BACKGROUND} \\ Although Heller myotomy is one of the most effective treatments for acha- \\ lasia, it may be associated with early or late symptom relapse in some patients. \\ Therefore, additional treatment is required to achieve better control of symp- \\ toms. \\ Aim: To evaluate the safety and efficacy of pneumatic balloon dilation \\ (PBD) in patients with symptom relapse after Heller myotomy.

\section{METHODS} \\ Thirty six post-myotomy patients were evaluated from 1993 to 2013 . Six \\ patients were excluded from the analysis because of comorbid diseases or epi- \\ phrenic diverticula. Thirty patients were treated with PBD. Primary outcome \\ was defined as a decrease in symptom score to 4 or less and a reduction greater \\ than $80 \%$ from the baseline in the volume of barium in timed barium esopha- \\ gogram in 6 weeks. Achalasia symptom score (ASS) was assessed at 1.5, 3, 6, \\ and 12 months after treatment and then every six months in all patients and PBD \\ was repeated in case of symptom relapse (ASS $>4$ ).

\section{RESULTS} \\ The mean age of the patients was $45.5 \pm 13.9$ years (range: $21-73$ ). Primary out- \\ come was observed in 25 patients $(83 \%)$. The mean ASS of the patients dropped \\ from 7.8 before treatment to $1.3 \pm 2.0$ at 1.5 months after treatment $(p=0.0001)$. \\ The mean volume and height of barium decreased from $43.1 \pm 33.4$ and $7.1 \pm 4.7$ \\ to $6.0 \pm 17.1$ and $1.1 \pm 2.2$, respectively $(p=0.003, p=0.003)$. The mean duration of \\ follow-up was $11.8 \pm 6.3$ years. At the end of the study, 21 patients $(70 \%)$ reported \\ sustained good response. No major complications such as perforation or gross \\ bleeding were seen.
}

\section{CONCLUSION}

PBD is an effective and safe treatment option for achalasia in patients with symptom relapse after Heller myotomy.

\section{KEYWORDS}

Achalasia; Pneumatic balloon dilatation; Myotomy

* Corresponding Author:

Javad Mikaeli, MD

Digestive Disease Research Institute, Shariati Hospital, Tehran University of Medical Sciences, Tehran, Iran

Tel: +982182415104

Fax: +982182415500

Email:mikaeli@ams.ac.ir

Received: 17 Jun. 2015

Accepted: 11 Nov. 2015
Please cite this paper as:

Amani M, Fazlollahi N, Shirani S, Malekzadeh R, Mikaeli J. Assessment of Pneumatic Balloon Dilation in Patients with Symptomatic Relapse after Failed Heller Myotomy: A Single Center Experience. Disease Middle East J Dig Dis 2015;8:57-62. DOI :10.15171/mejdd.2016.08

\section{INTRODUCTION}

Idiopathic achalasia is a chronic disease of the esophagus. Endoscopic and surgical methods of treatment aim to palliate the typical 
symptoms of this disease. ${ }^{1}$ Achalasia, however, is a recurrent disease with relapse rates reaching $25-30 \%$ in 5-year follow-up. ${ }^{2,3}$ Various types of surgery, such as open and laparoscopic Heller myotomy $(\mathrm{HM})^{2}$ and per-oral endoscopic myotomy, ${ }^{4}$ produce good responses in patients. However, some relapses, related to incomplete myotomy or sclerosis of the distal site of the myotomy, occur after surgery. ${ }^{5-8}$

The method for retreatment of patients who had relapsed after myotomy is controversial. Some studies have suggested pneumatic balloon dilation (PBD) for patients with symptomatic relapse after myotomy. ${ }^{5,6,9-11}$ In those studies, PBD has been shown to be safe and effective in short-term ${ }^{9,10,11}$ and long-term ${ }^{6}$ follow-up in the patients who had relapsed after myotomy, without increased risk of perforation. ${ }^{9,10,11}$

In this paper, we report our experience about the safety and long-term efficacy of PBD in patients who had symptom relapse after HM.

\section{MATERIALS AND METHODS}

We retrospectively evaluated all patients who underwent HM after failure of PBD to offer symptomatic relief over a period of 20 years from 1993 to 2013. The diagnosis of idiopathic achalasia was based on clinical symptoms as well as radiological and endoscopic findings and confirmed by manometric criteria. The exclusion criteria were a good response to $\mathrm{HM}$, poor cooperation, epiphrenic diverticula, and severe comorbid diseases. Subjects were also excluded if endoscopy revealed esophageal ulcers or esophageal varices.

Informed consent was obtained from all the patients after a full explanation of risks, benefits, and alternatives. The Ethics Committee for Medical Research at Tehran University of Medical Sciences reviewed and approved the study protocol.

Symptom scores were assessed in all the patients by a single physician, all timed barium esophagograms (TBEs) were done by a single radiologist, and all PBDs were performed by the same gastroenterologist.

\section{Symptomatic response}

A standard questionnaire was used for calculating the achalasia symptom score (ASS) as in our previous studies. ${ }^{12,13}$ The severity of each symptom was recorded on a scale of 0 to 3 , depending on its frequency. The total symptom score was the sum of five cardinal symptoms scores and the severity score of dysphagia (tables 1, and 2). Therefore, the highest possible score was 18. Regurgitation was defined as returning of food material from the esophagus, active in standing or sitting and passive in supine or lying positions. The ASS was calculated for each patient before treatment, 1.5, 3, 6, 9 and 12 months after treatment, then every 6 months, and finally at the end of the follow-up period. The patients were also requested to come back for re-evaluation if they felt severe dysphagia or regurgitation any time over the follow-up period. A good clinical response was defined as a decrease in ASS to 4 or less, while an increase of 2 or more points in the severity of dysphagia after the initial good response was considered as clinical relapse. A sustained good response was defined as remaining in clinical remission (ASS equal to or less than 4) at the end of the follow-up.

\section{Timed barium esophagogram}

All the patients underwent TBE as an objective tool to assess esophageal emptying before and 1.5 months after treatment. In the upright position, 200 $\mathrm{mL}$ of barium sulfate suspension ( $81 \%$ weight/volume) 14 was swallowed by the patients, and radiographies were done at 1,3 , and 5 minutes after swallowing from the left posterior oblique view. The barium column height, the distance from the most distal part of the esophagus to the most proximal barium level, was measured in centimeters. The volume of retained barium in milliliters was calculated as follows: (mean radius) ${ }^{2} \times 3.14 \times$ height of column. These calculations have been used in similar studies. ${ }^{12,13}$

The difference in the retained barium height and volume at 5 minutes was calculated between the pre-and post-treatment TBEs. We defined a good response as a reduction greater than $80 \%$ from the 


\begin{tabular}{lcccc}
\multicolumn{4}{l}{ Table 1: Cardinal symptoms score } & \\
\hline Symptom & Each meal & Daily & Weekly & None \\
\hline $\begin{array}{l}\text { Dysphagia } \\
\text { solids }\end{array}$ & 3 & 2 & 1 & 0 \\
$\begin{array}{l}\text { Dysphagia } \\
\text { liquids }\end{array}$ & 3 & 2 & 1 & 0 \\
$\begin{array}{l}\text { Active } \\
\text { regurgitation }\end{array}$ & 3 & 2 & 1 & 0 \\
$\begin{array}{l}\text { Symptom } \\
\begin{array}{l}\text { Passive } \\
\text { regurgitation }\end{array}\end{array}$ & 3 & 2 & 1 & 0 \\
Chest pain & 3 & 2 & 1 & 0 \\
\hline
\end{tabular}

\begin{tabular}{|c|c|c|}
\hline Severity & Score & Description \\
\hline No dysphagia & 0 & $\begin{array}{l}\text { Normal passage } \\
\text { of food from LES } \\
\text { zone }\end{array}$ \\
\hline Mild dysphagia & 1 & $\begin{array}{l}\text { Sensation or short } \\
\text { delay of passage } \\
\text { of food from LES, } \\
\text { without need for } \\
\text { water }\end{array}$ \\
\hline Modrate dysphagia & 2 & $\begin{array}{l}\text { Need for water for } \\
\text { passage of food } \\
\text { from LES zone }\end{array}$ \\
\hline Severe dysphagia & 3 & $\begin{array}{l}\text { Accompanied by } \\
\text { passive or active } \\
\text { regurgitation }\end{array}$ \\
\hline
\end{tabular}

LES: Lower esophageal sphincter

baseline in the height and volume of barium at 1.5 months after PBD, and a poor response as lack of a good TBE response.

\section{Manometry}

Achalasia had been confirmed by manometry in all the patients before HM. Twenty three of the enrolled patients had conventional manometry and were not classified into Chicago classification subtypes. Seven patients underwent high resolution manometry of whom six had type II, and one had type I. We did not repeat esophageal manometry before or after PBD to delineate the etiology of prospective symptomatic relapse.

\section{Pneumatic balloon dilation}

All PBDs were done using Rigiflex balloon (Boston Scientific Corporation, Natick, MA), under direct endoscopic vision with graded scale. After a clear liquid diet for $24 \mathrm{~h}$ and an overnight fast, the patients were sedated with intravenous diazepam (5-10 mg) and meperidine (25-50 mg). Following a complete upper gastrointestinal endoscopy, the balloon dilator was passed over guide wire and positioned such that the midpoint of the balloon was placed at the gastroesophageal junction (GEJ) under videoendoscopic guide. Rigiflex balloons of $30 \mathrm{~mm}$ were gradually inflated up to $15 \mathrm{psi}$ in $30 \mathrm{~s}$ and maintained for another $60 \mathrm{~s}$. After emptying and pulling out the balloon, the patients underwent endoscopy again for assessment of the lower esophageal sphincter opening (relaxation) and any evidence of bleeding or perforation. They were discharged if they were asymptomatic after a 6-hour observation. Response to treatment was evaluated at $1.5,3,6,9$, and 12 months, and then every 6 months. PBD was repeated with 3.5 and 4 $\mathrm{cm}$ balloons if the symptoms relapsed.

\section{Study end points}

The primary study end point was reduction in ASS and TBE values 6 weeks after PBD compared with the baseline values (short-term response). The secondary end point was defined as a sustained good response at the end of follow-up (long-term response).

\section{Statistical analysis}

The results were analyzed using SPSS software version 19 for Windows. Continuous variables were expressed as mean $\pm \mathrm{SD}$. Comparisons were performed using paired $t$ test. A P value of less than 0.05 was chosen for rejection of the null hypothesis.

\section{RESULTS}

A total of 75 patients were identified who were referred for operative management. Thirty six patients had symptom relapse after HM. Six patients who had comorbid diseases or epiphrenic diverticula were treated by botulinum toxin injection and were excluded from the study. Of the 30 patients who were enrolled in this study, seven had been treated by laparoscopic myotomy and the others by open thoracotomy. Fifteen patients were men and 15 were women with a mean age of $45.5 \pm 13.9$ years 


\begin{tabular}{|c|c|}
\hline Mean age (year) & $45.5 \pm 13.9$ \\
\hline \multirow[t]{2}{*}{ Sex } & 15 \\
\hline & 15 \\
\hline ASS before treatment & $7.8 \pm 3.4$ \\
\hline Mean IRP before treatment & $23.9 \pm 10.5 \mathrm{mmHg}$ \\
\hline
\end{tabular}

(range: 21-73, table 3). Age and sex did not correlate with response to treatment.

The mean symptom-presentation period after surgery was $6.7 \pm 7.1$ years (range: $1-20$ years). The mean duration of follow-up was $11.8 \pm 6.3$ years and none of the patients was lost to follow-up after treatment.

Overall, 51 balloon dilations were performed for the patients (mean 1.7 balloon for each patient), of them 30 with $3 \mathrm{~cm}, 17$ with $3.5 \mathrm{~cm}$, and 4 with $4 \mathrm{~cm}$ Rigiflex balloon. The mean duration of relapse after the first PBD was 8.2 \pm 7.2 months (2-26 months) and after the second PBD was $18.3 \pm 14.8$ months (range: 5-43 months). No severe complications (perforation or bleeding) were seen.

\section{Primary outcome after pneumatic dilatation}

Primary outcome was seen in 25 patients ( $83 \%$ ). The symptoms of 17 patients relapsed in a mean period of $8.2 \pm 7.2$ months (range: 2-26 months), for whom PBD with $3.5 \mathrm{~cm}$ Rigiflex balloon was performed. In four patients, relapse occurred after the second PBD, which required $4 \mathrm{~cm}$ balloon dilation.

The mean symptom score dropped from 7.8 $( \pm 3.4)$ to1.3 $( \pm 2.1)(p=0.0001$, figure 1$)$. In TBE, the mean retained barium volume at the 5 th minute was reduced from $43.1 \mathrm{~mL}( \pm 33.4)$ before treatment to $6.0 \mathrm{~mL}( \pm 17.1), 1.5$ months after treatment ( $p=0.003$, figure 2 ). The mean height of barium decreased from $7.1( \pm 4.7) \mathrm{cm}$ before treatment to $0.1( \pm 2.2) \mathrm{cm}(p=0.003) 1.5$ months after treatment (figure 3).

\section{Long-term outcome}

Eventually at the end of the follow-up, 21 patients $(70 \%)$ showed sustained good responses and 9 patients ( 3 men, 6 women) with mean age $36.9 \pm$
15.8 showed poor responses. However, there was no significant difference between sex and age in these two groups ( $p=0.3, p=0.1$ ).

The mean symptom score dropped from 7.8 $( \pm 3.4)$ to $3.1( \pm 2.6)(p=0.0001)$ at the end of the follow-up (figure 1). In TBE, the mean retained barium volume at the 5 th minute, decreased from $43.1 \mathrm{~mL}( \pm 33.4)$ to $2.8 \mathrm{~mL}( \pm 7.2)(p=0.004)$ at the end of the follow-up (figure 2). The mean height of barium decreased from $7.1( \pm 4.7)$ before treatment to $0.8( \pm 1.9)(p=0.002)$ at the end of the follow-up (figure 3).

\section{DISCUSSION}

In this retrospective study, we assessed the safety and efficacy of PBD in patients who had relapse after HM in the long-term follow-up. Our results revealed graded PBD as a safe method that can be used for retreatment after failure of myotomy.

Although short and medium-term outcomes after HM have been good, its effectiveness decreases over time and the relapse rates at 5-7 years after HM have ranged from $18 \%$ to $21 \% .{ }^{11}$ However, our observation revealed more relapses than previous studies with range of $29 \%$ to $37 \%$ at $5-10$ years.

In a recent study, Kumbhari and colleagues 9 reported that out of 27 patients with achalasia who had relapse after HM and received PBD, 24 had good responses after 12 months of follow-up. The median follow-up duration was 30 months. They had a total of 50 PBDs in 27 patients with no perforation. Finally, they concluded that following HM, PBD was safe and yielded an excellent short-term response rate. ${ }^{9}$ Zaninotto and co-workers treated nine patients with symptom relapse after HM by PBD. Good response to PBD (median 2 dilations) was seen in seven of the nine patients in a mean follow-up period of 14.5 months.5 In another study in 2004, among 139 patients (117 untreated and 22 failed HM) who were treated by PBD, two perforations occurred in previously untreated cases. The authors concluded that the PBD perforation risk did not increase after HM. ${ }^{10}$

In our study, PBDs were done for 30 patients who had post-myotomy symptom relapse after a mean 


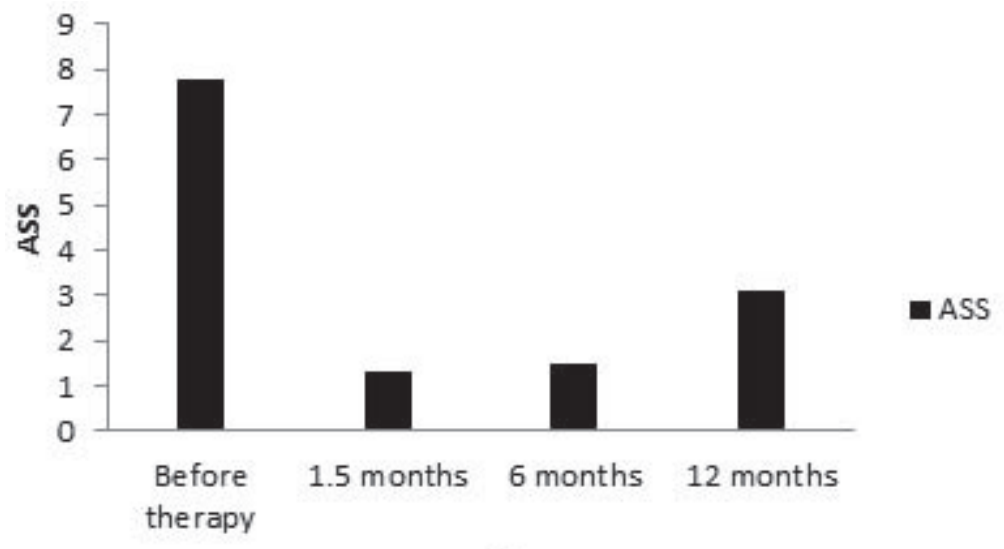

months

Fig. 1: Comparison of achalasia symptom score before and after treatment

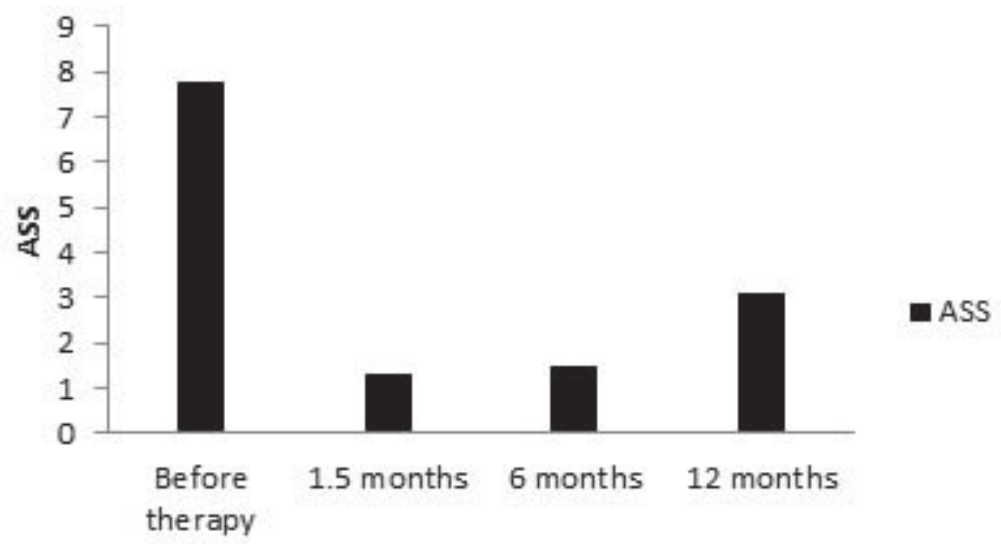

months

Fig. 2: Comparison of retained barium volume $(\mathrm{mL})$ in the 5 th minute of timed barium esophagogram before and after treatment

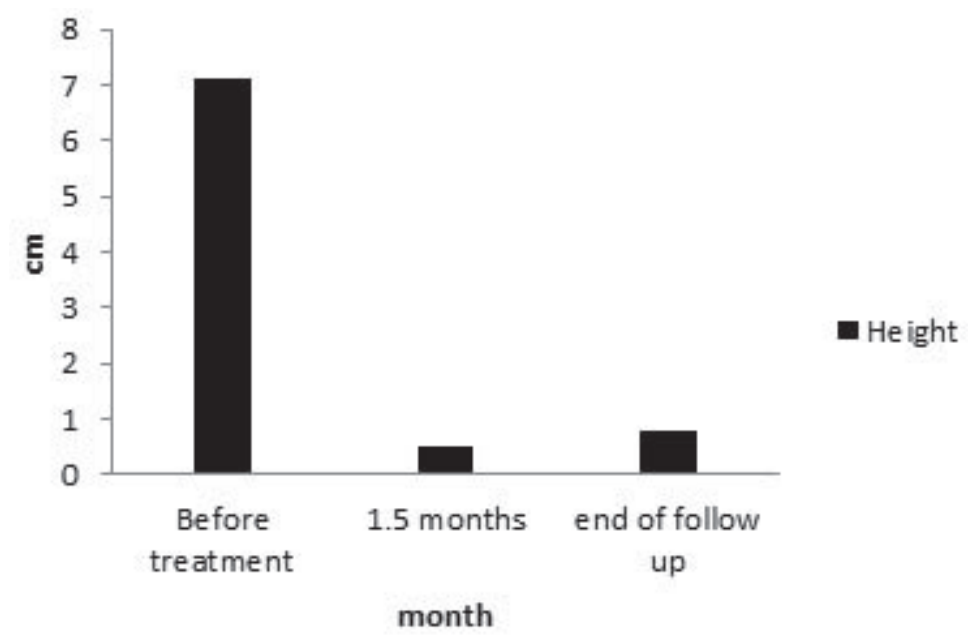

Fig. 3: Comparison of retained barium height $(\mathrm{cm})$, in the 5 th minute of timed barium esophagogram before and after treatment 
duration of $6.7 \pm 7.1$ years. Twenty five patients had good initial response $(83 \%)$ at 1.5 months after the first PBD. Overall, 51 balloon dilations were done for the patients in the follow-up period without any perforation. At the end of the study, sustained good response was reported in 21 patients (70\%).

In conclusion, graded pneumatic dilation from 30 up to $40 \mathrm{~mm}$ balloon diameter as needed, is a safe and effective method for treating idiopathic achalasia ${ }^{15,16}$ and it can be used for patients who report symptom relapse after myotomy. It seems that if graded balloon dilation is applied cautiously, the risk of esophageal perforation will not increase in patients after myotomy.

\section{CONFLICT OF INTEREST}

The authors declare no conflict of interest related to this work.

\section{REFERENCES}

1. Campos GM, Vittinghoff E, Rabl C, Takata M, Gadenstatter M, Lin F, et al. Endoscopic and surgical treatments for achalasia: a systematic review and meta-analysis. Ann Surg 2009;249:45-57. DOI:10.1097/SLA.0b013e31818e43ab.

2. Zerbib F, Thetiot V, Richy F, Benajah DA, Message L, Lamouliatte H. Repeated pneumatic dilations as longterm maintenance therapy for esophageal achalasia. Am $J$ Gastroenterol 2006;101:692-7. DOI:10.1111/j.15720241.2006.00385.x.

3. Vela MF, Richter JE, Wachsberger D, Connor J, Rice TW. Complexities of managing achalasia at a tertiary referral center: use of pneumatic dilatation, Heller myotomy, and botulinum toxin injection. Am J Gastroenterol 2004;99:102936. DOI:10.1111/j.1572-0241.2004.30199.x.

4. von Renteln D, Inoue H, Minami H, Werner YB, Pace A, Kersten JF, et al. Peroral endoscopic myotomy for the treatment of achalasia: a prospective single center study. Am J Gastroenterol 2012;107:411-7. DOI:10.1038/ ajg.2011.388.

5. Zaninotto G, Costantini M, Portale G, Battaglia G, Molena $\mathrm{D}$, Carta A, et al. Etiology, diagnosis, and treatment of failures after laparoscopic Heller myotomy for achalasia. Ann Surg 2002;235:186-92.

6. Legros L, Ropert A, Brochard C, Bouguen G, Pagenault M, Siproudhis L, et al. Long-term results of pneumatic dilatation for relapsing symptoms of achalasia after Heller myotomy. Neurogastroenterol Motil 2014;26:1248-55. DOI:10.1111/nmo.12380.

7. Costantini M, Zaninotto G, Guirroli E, Rizzetto C, Portale G, Ruol A, et al. The laparoscopic Heller-Dor operation remains an effective treatment for esophageal achalasia at a minimum 6-year follow-up. Surg Endosc 2005;19:34551. DOI:10.1007/s00464-004-8941-7.

8. Bonatti H, Hinder RA, Klocker J, Neuhauser B, Klaus A, Achem SR, et al. Long-term results of laparoscopic Heller myotomy with partial fundoplication for the treatment of achalasia. Am J Surg 2005;190:874-8. DOI:http://dx.doi. org/10.1016/j.amjsurg.2005.08.012.

9. Kumbhari V, Behary J, Szczesniak M, Zhang T, Cook IJ Efficacy and safety of pneumatic dilatation for achalasia in the treatment of post-myotomy symptom relapse. Am J Gastroenterol 2013;108:1076-81. DOI:10.1038/ ajg.2013.32.

10. Guardino JM, Vela MF, Connor JT, Richter JE. Pneumatic dilation for the treatment of achalasia in untreated patients and patients with failed Heller myotomy. J Clin Gastroenterol 2004;38:855-60.

11. Kumar A, Itha S. Long-term outcome of pneumatic dilation in the treatment of achalasia. Am J Gastroenterol 2005;100:2130-1; author reply 1. DOI:10.1111/j.15720241.2005.50395-3.x

12. Niknam R, Mikaeli J, Fazlollahi N, Mahmoudi L, Mehrabi N, Shirani S, et al. Ethanolamine oleate as a novel therapy is effective in resistant idiopathic achalasia. Dis Esophagus 2014;27:611-6. DOI:10.1111/dote.12122.

13. Niknam R, Mikaeli J, Mehrabi N, Mahmoudi L, Elahi E, Shirani S, et al. Ethanolamine oleate in resistant idiopathic achalasia: a novel therapy. Eur J Gastroenterol Hepatol 2011;23:1111-5. DOI:10.1097/MEG.0b013e328349647e.

14. Montazeri G, Nouri N, Estakhri A, Shirani S, Derakhshan MH, Yaghoobi M, et al. Lower oesophageal sphincter pressure and timed barium oesophagogram: two objective parameters in the non-invasive assessment of primary achalasia. Aliment Pharmacol Ther 2005;22:261-5. DOI:10.1111/j.1365-2036.2005.02557.x.

15. Mikaeli J YM, Sohrabi M, Malekzadeh R. Rigiflex Balloon dilation without fluoroscopy for treatment of Achalasia: A long-term follow-up of 99 patients. Acta Medica Iranica 2002;40:69-72.

16. Mikaeli J, Bishehsari F, Montazeri G, Yaghoobi M, Malekzadeh R. Pneumatic balloon dilatation in achalasia: a prospective comparison of safety and efficacy with different balloon diameters. Aliment Pharmacol Ther 2004;20:431-6. DOI:10.1111/j.1365-2036.2004.02080.x. 